\title{
Two New Antimicrobial Dimeric Compounds: Febrifuquinone, a Vismione-Anthraquinone Coupled Pigment and Adamabianthrone, from two Psorospermum Species
}

\author{
Maurice Tsaffack, ${ }^{a}$ Jean Robert Nguemeving, ${ }^{a}$ Victor Kuete, ${ }^{b}$ Basile Le Sage Ndejouong Tchize, \\ Pierre Mkounga, ${ }^{a}$ Véronique Penlap Beng, ${ }^{d}$ Philip Gregory Hultin, ${ }^{e}$ Etienne Tsamo, ${ }^{a}$ and \\ Augustin Ephrem NKENGFACK ${ }^{*, a}$ \\ ${ }^{a}$ Department of Organic Chemistry, TWAS Research Unit, Yaounde University I; ${ }^{d}$ Department of Biochemistry, Yaounde \\ University I; P. O. Box 812 Yaounde, Cameroon: ${ }^{b}$ Department of Biochemistry, Dschang University; P. O. Box 67 Dschang, \\ Cameroon: ${ }^{c}$ Department of Biomolecular Chemistry, Leibniz Institute for Natural Products Research and Infection \\ Biology; Beutenbergstrasse 11a, 07745 Jena, Germany: and ${ }^{e}$ Department of Chemistry, Manitoba University; Winnipeg, \\ MB R3T 2N2, Canada. Received March 31, 2009; accepted July 1, 2009; published online July 8, 2009
}

Febrifuquinone (1), a new vismione-anthraquinone coupled pigment and a new bianthrone named adamabianthrone (2), were isolated respectively, from the roots of Psorospermum febrifugum and from the bark of Psorospermum adamauense along with eight known compounds including: two bianthrones [(bianthrone $A_{1}(3)$ and bianthrone $A_{2 b}$ l, one vismione [(vismione D (4)], one anthrone (3-geranyloxyemodin anthrone) and four anthraquinones [(1,8-dihydroxy-3-isoprenyloxy-6-methylanthraquinone, emodin (5), 3-geranyloxy-1,8-dihydroxy-6methylanthraquinone and 2-geranyl-1,8-dihydroxy-6-methylanthraquinone]. Their structures were determined using modern spectroscopic methods including one and two dimensional-NMR techniques as well as MS. Compounds 1 and 2 showed significant antimicrobial activities against a wide range of bacteria and fungi.

Key words Psorospermum febrifugum; Psorospermum adamauense; quinone; antimicrobial test

The genus Psorospermum, belonging to the tribe Vismieae (family Guttiferae, subfamily Hypericoideae), comprises 55 species found in tropical Africa and Madasgascar. ${ }^{1)}$ Psorospermum species are shrub or small trees that are largely used in the African folk medicine as febrifugal, antidote against poison and purgative. They are also used as a remedy for the treatment of leprosy, skin diseases (such as dermatitis, scabies and eczemas) and subcutaneous wounds. ${ }^{2-4)}$ Previous chemical studies of different species of this genus have led to the isolation of vismiones, ${ }^{5-8)}$ anthraquinones, ${ }^{5,9)}$ anthrones and bianthrones, ${ }^{7,10-12)}$ xanthonolignoids ${ }^{13}$ and xanthones. $^{14-16)}$ On the other hand, the ethanolic extract of Psorospermum febrifugum was found to exhibit significant in vivo cytotoxicity and antitumor activity against the P-388 lymphocytic leukemia (3PS) in mice and in vitro, activity against a cell culture derived from human epidermoid carcinoma of the nasopharynx $(9 \mathrm{~KB}) .{ }^{15)}$ Further biological activity guided investigation, was undertaken ${ }^{14)}$ and led to the isolation of the first dihydrofuranoxanthone named psorospermin, which exhibited significant cytotoxicity and antitumor activity. ${ }^{16)}$ in addition to the other bioactive classes of secondary metabolites. Consequently, prompted by this result, the investigation on two Cameroonian Psorospermum species: Psorospermum febrifugum and Psorospermum adamauense was carried out. On Psorospermum adamauense, no phytochemical study has yet been reported to the best of our knowledge. The present work deals with the isolation, structure elucidation of secondary metabolites as well as the evaluation of their antimicrobial activities.

\section{Results and Discussion}

Air dried and powdered roots of $P$. febrifugum and bark of $P$. adamauense were extracted at room temperature with a mixture of methanol/methylenechloride (1:1), respectively. The residue, obtained from each plant after evaporation of the solvents, was respectively subjected to repeated silica gel column chromatography (see Experimental) and eluted with a mixture of hexane/AcOEt of increasing polarity. From the roots extract of $P$. febrifugum, four compounds were obtained among which, one new derivative named febrifuquinone (1) and three known compounds: bianthrone $\mathrm{A}_{1}(\mathbf{3})$, vismione $\mathrm{D}$ (4) and 3-geranyloxyemodin anthrone. While, from the bark of $P$. adamauense, one new compound named adamabianthrone (2), was isolated along with six known others:

3-Geranyloxyemodin anthrone, 1,8-dihydroxy-3-isoprenyloxy-6-methylanthraquinone, bianthrone $\mathrm{A}_{2 \mathrm{~b}}$, emodin (5), 3geranyloxy-1,8-dihydroxy-6-methylanthraquinone and 2-geranyl-1,8-dihydroxy-6-methylanthraquinone. The structures of the known compounds were determined from their ${ }^{1} \mathrm{H}$-, ${ }^{13} \mathrm{C}-\mathrm{NMR}$ and mass spectral data and confirmed by comparison of these data and their physical constants with those published in the literature. ${ }^{5-18)}$

Compound 1, named febrifuquinone, mp $132-133^{\circ} \mathrm{C}$, was isolated as red powder. It reacted positively to the $\mathrm{FeCl}_{3}$ reagent suggesting its phenolic nature. Its "high resolution electrospray ionization-time-of-flight (HR-ESI-TOF)" MS showed a protonated molecule $[\mathrm{M}+\mathrm{H}]^{+}$at $\mathrm{m} / \mathrm{z} 679.2514$ (Calcd for $\mathrm{C}_{40} \mathrm{H}_{39} \mathrm{O}_{10}: 679.2532$ ) corresponding to the molecular formula $\mathrm{C}_{40} \mathrm{H}_{38} \mathrm{O}_{10}$ with 22 degrees of unsaturation.

The broad band proton decoupled ${ }^{13} \mathrm{C}$-NMR spectrum (DMSO- $d_{6}$ ) of compound $\mathbf{1}$, revealed the presence of 40 carbon signals which were sorted by attached proton test (APT) and heteronuclear single quantum coherence (HSQC) techniques as five methyls, four methylenes, nine methines and twenty-two quaternary carbon atoms among which, three carbonyl groups at $\delta_{\mathrm{C}} 181.2, \delta_{\mathrm{C}} 189.6$ and $\delta_{\mathrm{C}} 203.6$ (see Table 1).

The UV spectrum of compound 1 showed absorption bands at 235, 278, 320, $390 \mathrm{~nm}$ and 408, $435 \mathrm{~nm}$ characteristic of vismione and 1,8-dihydroxyanthraquinone chromo- 
<smiles>CC(C)=CCCC(C)=CCOc1cc(C)c2c(C)c3c(cc2c1)C(c1c(C)cc(C)c2c1C(=O)c1c(O)cc(C)cc1C2=O)C(C)(C)CC3=O</smiles><smiles>COc1cc(O)c2c(c1)C(C1c3cc(C)cc(O)c3C(=O)c3c(O)cc(OC)cc31)c1cc(C)cc(O)c1C2=O</smiles>

phores. ${ }^{5,19)}$ These assumptions were supported by the ${ }^{1} \mathrm{H}$ NMR spectrum (DMSO- $d_{6}$ ) of compound 1 which showed two singlets, of one proton each, at $\delta_{\mathrm{H}} 9.78$ and $\delta_{\mathrm{H}} 15.79$ exchangeable with $\mathrm{D}_{2} \mathrm{O}$ and assigned to two hydroxyl groups, located, respectively at C-1 and C-9 of a vismione moiety ${ }^{20}$ and two other singlets of one proton each at $\delta_{\mathrm{H}} 11.99$ and $\delta_{\mathrm{H}}$ 12.06 also exchangeable with $\mathrm{D}_{2} \mathrm{O}$ and corresponding to two chelated hydroxyl groups at $\mathrm{C}-1$ and $\mathrm{C}-8$ of an anthraquinone moiety. ${ }^{19)}$ These data, combined with the protonated molecule $[\mathrm{M}+\mathrm{H}]^{+}$at $m / z 679.2514$, suggested that compound $\mathbf{1}$ is a dimmer containing in its skeleton, a vismione and an anthraquinone moieties.

This assumption was confirmed by the ${ }^{1} \mathrm{H}-\mathrm{NMR}$ spectrum (DMSO- $d_{6}$ ) of $\mathbf{1}$ which showed two sets of signals. The multiplicities and chemical shifts of the first set of signals were very close to those observed in the ${ }^{1} \mathrm{H}-\mathrm{NMR}$ spectrum of vismione $\mathrm{D}^{5)}(4)$ (see Table 1), while those of the second set, attributed to fragment "B," were quite similar to signals appeared in the ${ }^{1} \mathrm{H}-\mathrm{NMR}$ spectrum of emodin $(\mathbf{5})^{17)}$ (see also Table 1).

The junction between the two moieties was established using heteronuclear multiple bond correlation (HMBC) techniques (Fig. 1) which showed, ${ }^{2} J,{ }^{3} J$ correlations peaks between the proton singlet $\mathrm{H}-5\left(\delta_{\mathrm{H}} 2.96\right)$ belonging to vismione D moiety and C-5' $\left(\delta_{\mathrm{C}} 137.3\right), \mathrm{C}-6^{\prime}\left(\delta_{\mathrm{C}} 148.1\right)$ and C-10'a $\left(\delta_{\mathrm{C}} 108.3\right)$ belonging to emodin moiety. This result clearly indicated that the junction between the two moieties was through $\mathrm{C}-5$ and $\mathrm{C}-5^{\prime}$ linkage. On the other hand, the geranyloxy moiety was located at C-3 according to the cross peak observed on its nuclear Overhauser enhancement and exchange spectroscopy (NOESY) spectrum (Fig. 1) between the methylene proton at $\delta_{\mathrm{H}} 4.62$ and $\mathrm{H}-2\left(\delta_{\mathrm{H}} 6.38\right)$ and $\mathrm{H}-4$ $\left(\delta_{\mathrm{H}} 6.70\right)$.

This was further supported by the HR-ESI-TOF-MS/MS of the protonated molecule which showed the presence of two important fragment at $m / z 409\left[\mathrm{M}-\mathrm{C}_{15} \mathrm{H}_{9} \mathrm{O}_{5}\right]^{+}$(of vis-<smiles>CC(C)=CCC/C(C)=C/COc1cc(O)c2c(c1)C(C1c3cc(I)cc(O)c3C(=O)c3c(O)cc(I)cc31)c1cc(O)c(CC=C(C)C)c(O)c1C2=O</smiles><smiles>Cc1cc(O)c2c(c1)C(=O)c1cc(O)cc(O)c1C2=O</smiles>

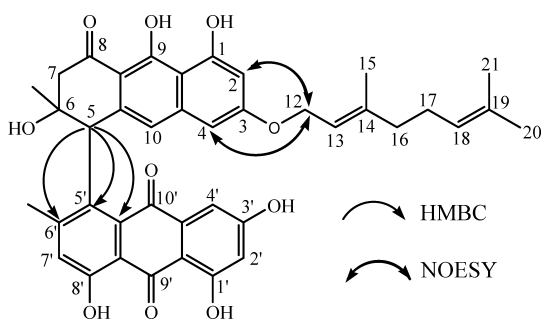

Fig. 1. Structure, Key HMBC and NOESY Correlations of Compound 1

mione D fragment) and $m / z 269\left[\mathrm{M}-\mathrm{C}_{25} \mathrm{H}_{29} \mathrm{O}_{5}\right]^{+}$(of emodin fragment) resulting from the breaking of the C-5, C-5' bond. On the basis of the above results, compound $\mathbf{1}$ was identified as 1-(6-((E)-3,7-dimethylocta-2,6-dienyloxy)-1,2,3,4-tetrahydro-3,8,9-trihydroxy-3-methyl-1-oxoanthracen-4-yl)-4,5,7trihydroxy-2-methylanthracene-9,10-dione named febrifuquinone (see Fig. 1).

Adamabianthrone (2), mp $152-154^{\circ} \mathrm{C}$, was obtained from the bark of $P$. adamauense as a yellow powder in a mixture of hexane/ethylacetate $(1: 9)$. It reacted positively to the $\mathrm{FeCl}_{3}$ reagent and magnesium acetate, indicating the presence in its structure of phenolic hydroxyl groups and 1,8-dihydroxyanthrone skeleton, respectively. The molecular formula of compound 2 was determined as $\mathrm{C}_{45} \mathrm{H}_{46} \mathrm{O}_{8}$ from its "high resolution ESI-TOF (HR-ESI-TOF)" MS, which showed a protonated molecule $[\mathrm{M}+\mathrm{H}]^{+}$at $m / z$ 715.3231. The UV and ${ }^{1} \mathrm{H}-$ NMR spectra of $\mathbf{2}$ were very similar to those recorded for bianthrone $\mathrm{A}_{1}{ }^{10)}(\mathbf{3})$.

The major difference between the two compounds appeared in the ${ }^{1} \mathrm{H}-\mathrm{NMR}$ spectrum (DMSO- $d_{6}$ ) of compound 2 which, showed one set of signals $\left[\left(\delta_{\mathrm{H}} 1.60(3 \mathrm{H}, \mathrm{s}), \delta_{\mathrm{H}} 1.62\right.\right.$ $(3 \mathrm{H}, \mathrm{s})$ and $\delta_{\mathrm{H}} 1.81(3 \mathrm{H}, \mathrm{s}) ; \delta_{\mathrm{H}} 2.05(4 \mathrm{H}, \mathrm{m}) ; \delta_{\mathrm{H}} 4.64(2 \mathrm{H}, \mathrm{d}$, $J=6.0 \mathrm{~Hz}) / \delta_{\mathrm{C}} 68.2 ; \delta_{\mathrm{H}} 5.30(1 \mathrm{H}, \mathrm{t}, J=8 \mathrm{~Hz})$ and $\delta_{\mathrm{H}} 5.46$ $\left.(1 \mathrm{H}, \mathrm{t}, J=6.0 \mathrm{~Hz}) / \delta_{\mathrm{C}} 119.3\right]$, corresponding to one $O$-geranyloxy group (instead of two, as in bianthrone $A_{1}(3)$ ) and an- 
Table 1. ${ }^{1} \mathrm{H}-\mathrm{NMR}$ Data of Compounds $\mathbf{1}, \mathbf{4}$ and $\mathbf{5}$ and ${ }^{13} \mathrm{C}-\mathrm{NMR}$ Data of Compound $\mathbf{1}$

\begin{tabular}{|c|c|c|c|c|}
\hline \multirow{2}{*}{ Position } & \multicolumn{2}{|c|}{1 DMSO- $d_{6}$} & \multirow{2}{*}{$\frac{4^{16)} \mathrm{CDCl}_{3}}{\delta_{\mathrm{H}}(\text { mult. } J \mathrm{~Hz})}$} & \multirow{2}{*}{$\frac{5^{17)} \text { Acetone- } d_{6}}{\delta_{\mathrm{H}}(\text { mult. } J \mathrm{~Hz})}$} \\
\hline & $\delta_{\mathrm{H}}($ mult. $J \mathrm{~Hz})$ & $\delta_{\mathrm{C}}(\mathrm{APT})$ & & \\
\hline $1-\mathrm{OH}$ & $9.78(1 \mathrm{H}, \mathrm{s})$ & $158.7(\mathrm{~s})$ & $9.79(1 \mathrm{H}, \mathrm{s})^{c)}$ & - \\
\hline 2 & $6.38(1 \mathrm{H}, \mathrm{d}, 2.2 \mathrm{~Hz})$ & $107.8(\mathrm{~d})$ & $6.49(1 \mathrm{H}, \mathrm{d}, 2.3 \mathrm{~Hz})$ & - \\
\hline 3 & - & $161.6(\mathrm{~s})$ & - & - \\
\hline 4 & $6.70(1 \mathrm{H}, \mathrm{d}, 2.2 \mathrm{~Hz})$ & $100.7(d)$ & $6.44(1 \mathrm{H}, \mathrm{d}, 2.3 \mathrm{~Hz})$ & - \\
\hline $4 a$ & - & $135.5(\mathrm{~s})$ & - & - \\
\hline 5 & $2.96(1 \mathrm{H}, \mathrm{s})$ & $42.4(\mathrm{~d})$ & $3.03(2 \mathrm{H}$, br s $)$ & - \\
\hline $6-\mathrm{OH}$ & $4.30(1 \mathrm{H}, \mathrm{s})$ & $69.4(\mathrm{~s})$ & - & - \\
\hline 7 & $\begin{array}{l}2.65\left(1 \mathrm{H}, \mathrm{d}, 17 \mathrm{~Hz}, \mathrm{H}_{7 \mathrm{a}}\right)^{a)} \\
2.88\left(1 \mathrm{H}, \mathrm{d}, 17 \mathrm{~Hz}, \mathrm{H}_{7 \mathrm{~b}}\right)^{a)}\end{array}$ & $50.7(t)$ & $2.82(2 \mathrm{H}$, br s $)$ & - \\
\hline 8 & - & $203.6(\mathrm{~s})$ & - & - \\
\hline $8 \mathrm{a}$ & - & $108.3(\mathrm{~s})$ & - & - \\
\hline 9-OH & $15.79(1 \mathrm{H}, \mathrm{s})$ & $164.3(\mathrm{~s})$ & $16.12(1 \mathrm{H}, \mathrm{s})^{c)}$ & - \\
\hline $9 \mathrm{a}$ & - & $108.3(\mathrm{~s})$ & - & - \\
\hline 10 & $6.89(1 \mathrm{H}, \mathrm{s})$ & $116.7(\mathrm{~d})$ & $6.83(1 \mathrm{H}, \mathrm{s})$ & - \\
\hline $10 \mathrm{a}$ & - & $108.9(\mathrm{~s})$ & - & - \\
\hline 11 & $1.23(3 \mathrm{H}, \mathrm{s})$ & $28.8(q)$ & $1.44(3 \mathrm{H}, \mathrm{s})$ & - \\
\hline 12 & $4.62(2 \mathrm{H}, \mathrm{d}, 6.4 \mathrm{~Hz})$ & $64.6(t)$ & $4.62(2 \mathrm{H}, \mathrm{d}, 6.6 \mathrm{~Hz})$ & - \\
\hline 13 & $5.45(1 \mathrm{H}, \mathrm{t}, 6.4 \mathrm{~Hz})$ & $119.2(\mathrm{~d})$ & $5.50(1 \mathrm{H}, \mathrm{t}, 6.6 \mathrm{~Hz})$ & - \\
\hline 14 & - & $140.7(\mathrm{~s})$ & - & - \\
\hline 15 & $2.05(4 \mathrm{H}, \mathrm{m})$ & $38.8(\mathrm{t})$ & $2.11(4 \mathrm{H}, \mathrm{m})$ & - \\
\hline 16 & & $25.7(\mathrm{t})$ & & - \\
\hline 17 & $5.06(1 \mathrm{H}, \mathrm{t}, 7.5 \mathrm{~Hz})$ & $123.6(\mathrm{~d})$ & $5.30(1 \mathrm{H}, \mathrm{t}, 8 \mathrm{~Hz})$ & - \\
\hline 18 & - & $130.9(\mathrm{~s})$ & - & - \\
\hline 19 & $1.72(3 \mathrm{H}, \mathrm{s})$ & $16.3(q)$ & $1.75(3 \mathrm{H}, \mathrm{s})$ & - \\
\hline 20 & $1.56(3 \mathrm{H}, \mathrm{s})^{b)}$ & $17.4(q)$ & $\left.1.69(3 \mathrm{H}, \mathrm{s})^{a}\right)$ & - \\
\hline 21 & $1.60(3 \mathrm{H}, \mathrm{s})^{b)}$ & $25.3(q)$ & $\left.1.60(3 \mathrm{H}, \mathrm{s})^{a}\right)$ & - \\
\hline $1^{\prime}-\mathrm{OH}$ & $12.06(1 \mathrm{H}, \mathrm{s})^{a, c)}$ & $164.6(\mathrm{~s})$ & - & $12.15(1 \mathrm{H}, \mathrm{s})^{a, c)}$ \\
\hline $2^{\prime}$ & $6.57(1 \mathrm{H}, \mathrm{d}, 2.5 \mathrm{~Hz})$ & $108.6(d)$ & - & $6.66(1 \mathrm{H}, \mathrm{d}, 2.5 \mathrm{~Hz})$ \\
\hline $3^{\prime}-\mathrm{OH}$ & $11.33(1 \mathrm{H}, \mathrm{s})^{c)}$ & $165.4(\mathrm{~s})$ & - & - \\
\hline $4^{\prime}$ & $7.11(1 \mathrm{H}, \mathrm{d}, 2.5 \mathrm{~Hz})$ & $120.4(\mathrm{~d})$ & - & $7.25(1 \mathrm{H}, \mathrm{d}, 2.5 \mathrm{~Hz})$ \\
\hline $4^{\prime} \mathrm{a}$ & - & $135.0(\mathrm{~s})$ & - & - \\
\hline $5^{\prime}$ & - & $137.3(\mathrm{~s})$ & - & $7.58(1 \mathrm{H}, \mathrm{s})$ \\
\hline $6^{\prime}$ & - & $148.1(\mathrm{~s})$ & - & - \\
\hline $7^{\prime}$ & $6.77(1 \mathrm{H}, \mathrm{s})$ & $124.0(\mathrm{~d})$ & - & $7.15(1 \mathrm{H}, \mathrm{s})$ \\
\hline $8^{\prime}-\mathrm{OH}$ & $11.99(1 \mathrm{H}, \mathrm{s})^{a, c)}$ & $161.3(\mathrm{~s})$ & - & $12.05(1 \mathrm{H}, \mathrm{s})^{a, c)}$ \\
\hline $8^{\prime} a$ & - & $113.3(\mathrm{~s})$ & - & - \\
\hline $9^{\prime}$ & - & $189.6(\mathrm{~s})$ & - & - \\
\hline $9^{\prime} \mathrm{a}$ & - & $132.7(\mathrm{~s})$ & - & - \\
\hline $10^{\prime}$ & - & $181.2(\mathrm{~s})$ & - & - \\
\hline $10^{\prime} \mathrm{a}$ & - & $108.3(\mathrm{~s})$ & - & - \\
\hline $11^{\prime}$ & $2.40(3 \mathrm{H}, \mathrm{s})$ & $21.4(q)$ & - & $2.47(3 \mathrm{H}, \mathrm{s})$ \\
\hline
\end{tabular}

$a, b)$ Assignments bearing the same superscript in the same column may be reversed. $c$ ) Exchangeable in $\mathrm{D}_{2} \mathrm{O} .{ }^{1} \mathrm{H}-\mathrm{NMR}$ was measured at $400 \mathrm{MHz}$ for $\mathbf{1}$, at $200 \mathrm{MHz}$ for $\mathbf{4}$ and at $300 \mathrm{MHz}$ for $\mathbf{5} .{ }^{13} \mathrm{C}-\mathrm{NMR}$ of $\mathbf{1}$ was measured at $100 \mathrm{MHz}$.

other set of signals $\left[\left(\delta_{\mathrm{H}} 3.35(2 \mathrm{H}, \mathrm{d}, J=7.9 \mathrm{~Hz}) / \delta_{\mathrm{C}} 22.7, \delta_{\mathrm{H}}\right.\right.$ $5.10(1 \mathrm{H}, \mathrm{t}, J=7.9 \mathrm{~Hz}) / \delta_{\mathrm{C}} 123.9$ and $\left.\delta_{\mathrm{H}} 1.86(3 \mathrm{H}, \mathrm{s})\right]$, establishing the presence of a $\gamma, \gamma$-dimethylallyl moiety, in addition to a phenolic hydroxyl proton at $\delta_{\mathrm{H}} 9.54$ exchangeable with $\mathrm{D}_{2} \mathrm{O}$ (see Table 2). The absence in the ${ }^{1} \mathrm{H}-\mathrm{NMR}$ spectrum of adamabianthrone (2) of $\mathrm{H}-2^{\prime}$ signal (which appeared at $\delta_{\mathrm{H}} 6.35(1 \mathrm{H}, \mathrm{d}, J=2.2 \mathrm{~Hz})$ in bianthrone $\left.\mathrm{A}_{1}(3)\right)$, indicated that the $\gamma, \gamma$-dimethylallyl group was located at $\mathrm{C}-2^{\prime}$. This structure was further supported by analysis of the HMBC spectrum of compound 2 (Fig. 2) which showed cross peaks between the proton at $\delta_{\mathrm{H}} 3.35$ and carbons C-1' $\left(\delta_{\mathrm{C}} 161.8\right)$, $\mathrm{C}-2^{\prime}\left(\delta_{\mathrm{C}} 136.0\right)$ and $\mathrm{C}-3^{\prime}\left(\delta_{\mathrm{C}} 162.6\right)$ indicating that the $\gamma, \gamma-$ dimethylallyl group is located at $\mathrm{C}-2^{\prime}$. On the other hand, the geranyloxy moiety was located at $\mathrm{C}-3$, according to the cross peak observed on its NOESY spectrum (Fig. 2) between the methylene proton at $\delta_{\mathrm{H}} 4.64$ and $\mathrm{H}-2\left(\delta_{\mathrm{H}} 6.10\right)$ and $\mathrm{H}-4\left(\delta_{\mathrm{H}}\right.$ 6.30).

The relative stereochemistry of $\mathrm{H}-10$ and $\mathrm{H}-10^{\prime}$ was not

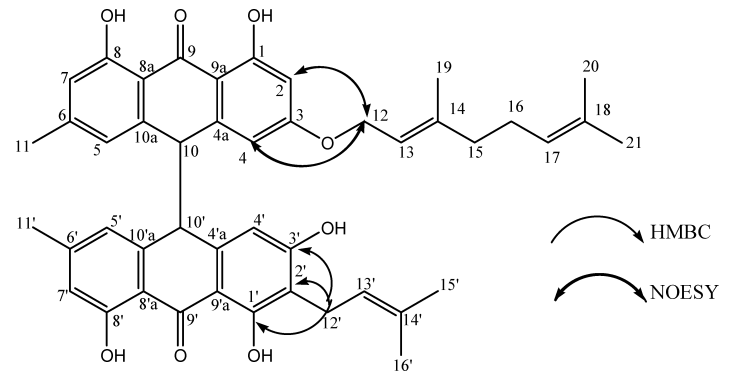

Fig. 2. Structure, Key HMBC and NOESY Correlations of Compound 2

determined. But, from the chemical shifts of the chelated hydroxyl groups at $\mathrm{C}-1 / \mathrm{C}-1^{\prime}\left(\delta_{\mathrm{H}} 12.37 / \delta_{\mathrm{H}} 12.05\right)$ and $\mathrm{C}-8 / \mathrm{C}-8^{\prime}$ $\left(\delta_{\mathrm{H}} 11.84 / \delta_{\mathrm{H}} 11.74\right)$ and also from those of the methyl groups at $\mathrm{C}-6 / \mathrm{C}-6^{\prime}\left(\delta_{\mathrm{H}} 2.25\right)$, we can confirmed on the basis of literature data, ${ }^{10)}$ that compound $\mathbf{2}$ was a mixture of meso and ( \pm )-racemic diastereoisomers. In fact, when only one of 
Table 2. ${ }^{1} \mathrm{H}-\mathrm{NMR}$ Data of Compounds 2 and 3 and ${ }^{13} \mathrm{C}-\mathrm{NMR}$ Data of Compound 2

\begin{tabular}{|c|c|c|c|}
\hline \multirow{2}{*}{ Position } & \multicolumn{2}{|c|}{2 DMSO- $d_{6}$} & \multirow{2}{*}{$\frac{\mathbf{3}^{27)} \quad \mathrm{CDCl}_{3}}{\delta_{\mathrm{H}}(\text { mult. } J \mathrm{~Hz})}$} \\
\hline & $\delta_{\mathrm{H}}($ mult. $J \mathrm{~Hz})$ & $\delta_{\mathrm{C}}(\mathrm{APT})$ & \\
\hline $1-\mathrm{OH}$ & $12.37(1 \mathrm{H}, \mathrm{s})^{a, c)}$ & $4.9(\mathrm{~s})$ & $12.10(1 \mathrm{H}, \mathrm{s})^{a, c)}$ \\
\hline 2 & $6.10(1 \mathrm{H}, \mathrm{d}, 2.5 \mathrm{~Hz})$ & $102.5(\mathrm{~d})$ & $6.35(1 \mathrm{H}, \mathrm{d}, 2.2 \mathrm{~Hz})$ \\
\hline 3 & - & $162.9(\mathrm{~s})$ & - \\
\hline 4 & $6.30(1 \mathrm{H}, \mathrm{d}, 2.5 \mathrm{~Hz})$ & $109.8(d)$ & $6.00(1 \mathrm{H}, \mathrm{d}, 2.2 \mathrm{~Hz})$ \\
\hline $4 a$ & - & $137.5(\mathrm{~s})$ & - \\
\hline 5 & $6.60(1 \mathrm{H}, \mathrm{d}, 1.8 \mathrm{~Hz})$ & $123.2(\mathrm{~d})$ & $6.12(1 \mathrm{H}$, br s $)$ \\
\hline 6 & - & $147.3(\mathrm{~s})$ & - \\
\hline 7 & $6.20(1 \mathrm{H}, \mathrm{d}, 1.8 \mathrm{~Hz})$ & $118.0(\mathrm{~d})$ & $6.69(1 \mathrm{H}$, br s $)$ \\
\hline $8-\mathrm{OH}$ & $11.84(1 \mathrm{H}, \mathrm{s})^{a, c)}$ & $162.8(\mathrm{~s})$ & $11.90(1 \mathrm{H}, \mathrm{s})^{a, c)}$ \\
\hline $8 \mathrm{a}$ & - & $112.9(\mathrm{~s})$ & - \\
\hline 9 & - & $190.7(\mathrm{~s})$ & - \\
\hline $9 \mathrm{a}$ & - & $111.9(\mathrm{~s})$ & - \\
\hline 10 & $4.50(1 \mathrm{H}, \mathrm{s})$ & $59.0(\mathrm{~d})$ & $4.33(1 \mathrm{H}, \mathrm{s})$ \\
\hline $10 \mathrm{a}$ & - & $139.9(\mathrm{~s})$ & - \\
\hline 11 & $2.25(3 \mathrm{H}, \mathrm{s})$ & $24.0(\mathrm{q})$ & $2.31(3 \mathrm{H}, \mathrm{s})$ \\
\hline 12 & $4.64(2 \mathrm{H}, \mathrm{d}, 6.0 \mathrm{~Hz})$ & $68.2(\mathrm{t})$ & $4.54(2 \mathrm{H}, \mathrm{d}, 6.6 \mathrm{~Hz})$ \\
\hline 13 & $5.46(1 \mathrm{H}, \mathrm{t}, 6.0 \mathrm{~Hz})$ & $119.3(d)$ & $5.47(1 \mathrm{H}, \mathrm{t}, 6.6 \mathrm{~Hz})$ \\
\hline 14 & - & $138.9(\mathrm{~s})$ & - \\
\hline 15 & $2.05(4 \mathrm{H}, \mathrm{m})$ & $39.6(\mathrm{t})$ & $2.12(2 \mathrm{H}, \mathrm{m})$ \\
\hline 16 & & $28.4(\mathrm{t})$ & $2.14(2 \mathrm{H}, \mathrm{m})$ \\
\hline 17 & $5.30(1 \mathrm{H}, \mathrm{t}, 8 \mathrm{~Hz})$ & $123.3(\mathrm{~d})$ & $5.12(1 \mathrm{H}, \mathrm{m})$ \\
\hline 18 & - & $131.9(\mathrm{~s})$ & - \\
\hline 19 & $1.81(3 \mathrm{H}, \mathrm{s})$ & $18.2(q)$ & $1.79(3 \mathrm{H}$, br s $)$ \\
\hline 20 & $\left.1.60(3 \mathrm{H}, \mathrm{s})^{b}\right)$ & $19.4(q)$ & $1.70(3 \mathrm{H}, \text { br s })^{b)}$ \\
\hline 21 & $1.62(3 \mathrm{H}, \mathrm{s})^{b)}$ & $27.1(q)$ & $1.63(3 \mathrm{H}, \text { br s })^{b)}$ \\
\hline $1^{\prime}-\mathrm{OH}$ & $12.05(1 \mathrm{H}, \mathrm{s})^{c, d)}$ & $161.8(\mathrm{~s})$ & $12.10(1 \mathrm{H}, \mathrm{s})^{c, d)}$ \\
\hline $2^{\prime}$ & - & $136.0(\mathrm{~s})$ & $6.35(1 \mathrm{H}, \mathrm{d}, 2.2 \mathrm{~Hz})$ \\
\hline $3^{\prime}-\mathrm{OH}$ & $9.54(1 \mathrm{H}, \mathrm{s})$ & $162.6(\mathrm{~s})$ & - \\
\hline $4^{\prime}$ & $6.27(1 \mathrm{H}, \mathrm{s})$ & $108.6(d)$ & $6.00(1 \mathrm{H}, \mathrm{d}, 2.2 \mathrm{~Hz})$ \\
\hline $4^{\prime} \mathrm{a}$ & - & $135.1(\mathrm{~s})$ & - \\
\hline $5^{\prime}$ & $6.66(1 \mathrm{H}, \mathrm{d}, 2.5 \mathrm{~Hz})$ & $122.4(\mathrm{~d})$ & $6.12(1 \mathrm{H}$, br s $)$ \\
\hline $6^{\prime}$ & - & $148.0(\mathrm{~s})$ & - \\
\hline $7^{\prime}$ & $6.37(1 \mathrm{H}, \mathrm{d}, 2.5 \mathrm{~Hz})$ & $117.6(d)$ & $6.69(1 \mathrm{H}$, br s $)$ \\
\hline $8^{\prime}-\mathrm{OH}$ & $11.74(1 \mathrm{H}, \mathrm{s})^{c, d)}$ & $162.6(\mathrm{~s})$ & $11.90(1 \mathrm{H}, \mathrm{s})^{c, d)}$ \\
\hline $8^{\prime} \mathrm{a}$ & - & $113.0(\mathrm{~s})$ & - \\
\hline $9^{\prime}$ & - & $190.3(\mathrm{~s})$ & - \\
\hline $9^{\prime} \mathrm{a}$ & - & $111.6(\mathrm{~s})$ & - \\
\hline $10^{\prime}$ & $4.50(1 \mathrm{H}, \mathrm{s})$ & $59.1(\mathrm{~d})$ & $4.33(1 \mathrm{H}, \mathrm{s})$ \\
\hline $10^{\prime} \mathrm{a}$ & - & $143.5(\mathrm{~s})$ & - \\
\hline $11^{\prime}$ & $2.25(3 \mathrm{H}, \mathrm{s})$ & $24.2(q)$ & $2.31(3 \mathrm{H}, \mathrm{s})$ \\
\hline $12^{\prime}$ & $3.35(2 \mathrm{H}, \mathrm{d}, 7.9 \mathrm{~Hz})$ & $22.7(\mathrm{t})$ & - \\
\hline $13^{\prime}$ & $5.10(1 \mathrm{H}, \mathrm{t}, 7.9 \mathrm{~Hz})$ & $123.9(\mathrm{~d})$ & - \\
\hline $14^{\prime}$ & - & $133.5(\mathrm{~s})$ & - \\
\hline $15^{\prime}$ & $1.86(3 \mathrm{H}, \mathrm{s})^{e)}$ & $20.4(q)$ & - \\
\hline $16^{\prime}$ & $1.86(3 \mathrm{H}, \mathrm{s})^{e)}$ & $27.1(q)$ & - \\
\hline
\end{tabular}

$a, b, c, d, e)$ Assignments bearing the same superscript in the same column may be reversed, $c$ ) Exchangeable in $\mathrm{D}_{2} \mathrm{O} .{ }^{1} \mathrm{H}-\mathrm{NMR}$ was measured at $400 \mathrm{MHz}$ for 2 and $3 .{ }^{13} \mathrm{C}$ NMR of 2 was measured at $100 \mathrm{MHz}$ those two forms is obtained, the chelated hydroxyl groups at $\mathrm{C}-1 / \mathrm{C}-1^{\prime}$ and $\mathrm{C}-8 / \mathrm{C}-8^{\prime}$ appears as a singlet of one proton. In the meso forms, chelated hydroxyl groups at $\mathrm{C}-1 / \mathrm{C}-1^{\prime}$ and $\mathrm{C}$ $8 / \mathrm{C}-8^{\prime}$ resonated at $\delta_{\mathrm{H}} 11.86$ and $\delta_{\mathrm{H}} 11.78$, respectively, ${ }^{10)}$ and in the ( \pm -racemic forms, they appears at $\delta_{\mathrm{H}} 11.92$ and $\delta_{\mathrm{H}} 11.68$, respectively. ${ }^{10)}$

From the above results, the structure of compound $\mathbf{2}$ was determined as 9-(3-((E)-3,7-dimethylocta-2,6-dienyloxy)9,10-dihydro-1,8-dihydroxy-6-methyl-9-oxoanthracen-10yl)-2,4,5-trihydroxy-7-methyl-3-(3-methylbut-2-enyl)anthracen-10(9H)-one, named adamabianthrone (see Fig. 2).

The two plants were tested for their antimicrobial activities against a wide range of microorganisms, including five Gram-negative bacteria, three Gram-positive bacteria and two yeasts. From the results obtained (Table 3), compound 1 showed significant activity on all the tested microorganisms, Streptococcus faecalis and Bacillus cereus being the most sensitive pathogens. The minimal inhibition concentration (MIC) values obtained with compound $\mathbf{1}$ varied from $14.37 \mu \mathrm{M}$ (on S. faecalis and B. cereus) to $230.03 \mu \mathrm{M}$ (on Microsporum audouinii and Citrobacter freundii). Compound 2 also exhibited a very good activity on 9 of the 10 $(90 \%)$ tested microorganisms. The lowest MIC values (27.34 $\mu \mathrm{M})$ observed with this compound was recorded against $S$. faecalis. Despite the fact that the MIC values obtained with the two tested compounds are greater than that of the reference antibiotics $(0.45-3.60 \mu \mathrm{M})$, their inhibitory potential could be considered as important, taking into account that some of the tested microorganisms were resistant to the commonly used antibiotics. Besides, the MIC values obtained, the tested compounds are generally four fold lesser than their corresponding minimal microbicidal concentration (MMC) values (Table 3 ). This indicates that the microbicidal effects of the tested compounds could be expected on the tested microorganisms. $^{21-25)}$

\section{Experimental}

General All melting points were measured on a Büchi apparatus and are uncorrected. The UV spectra were obtained on a Kontron Uvikon 932 spectrophotometer. The IR spectra were recorded on a Nicolet Impact 400D FT-IR spectrometer. The ${ }^{1} \mathrm{H}$ - and ${ }^{13} \mathrm{C}-\mathrm{NMR}$ data [including HSQC, HMBC, NOESY and ${ }^{1} \mathrm{H}-{ }^{1} \mathrm{H}$ COSY (correlation spectroscopy experiment)] were measured on a Bruker DRX-400 instrument operating at $400 \mathrm{MHz}$ for ${ }^{1} \mathrm{H}$ and $100 \mathrm{MHz}$ for ${ }^{13} \mathrm{C}$. The chemical shifts $(\delta)$ are reported in ppm downfield from tetramethylsilane (TMS) using TMS or the solvent signal as standard. The HR-ESI-TOF-MS and HR-ESI-TOF-MS/MS were obtained in the posi-

Table 3. Antimicrobial Activity of Compounds 1, 2 and Reference Antibiotics (RA)

\begin{tabular}{|c|c|c|c|c|c|c|}
\hline \multirow{2}{*}{ Microorganisms } & \multicolumn{3}{|c|}{$\begin{array}{l}\text { Minimum inhibition concentration in } \mu \mathrm{g} / \mathrm{ml} \text { and } \mu \mathrm{M} \\
\text { (in parenthesis) of the tested samples }\end{array}$} & \multicolumn{3}{|c|}{$\begin{array}{l}\text { Minimum microbicidal concentration in } \mu \mathrm{g} / \mathrm{ml} \\
\text { and } \mu \mathrm{M} \text { (in parenthesis) of the tested samples }\end{array}$} \\
\hline & 1 & 2 & $\left.\mathrm{RA}^{a}\right)$ & 1 & 2 & $\left.\mathrm{RA}^{a}\right)$ \\
\hline \multicolumn{7}{|c|}{ Gram-negative bacteria } \\
\hline C. freundii & $156.25(230.03)$ & $156.25(218.74)$ & $9.76(1.80)$ & $>156.25(>230.03)$ & $>156.25(>218.74)$ & $19.53(3.60)$ \\
\hline E. coli & $19.53(28.75)$ & $156.25(218.74)$ & $9.76(1.80)$ & $78.12(115.02)$ & $>156.25(>218.74)$ & $19.53(3.60)$ \\
\hline P. aeruginosa & $39.06(57.51)$ & $39.06(54.68)$ & $19.53(3.60)$ & $78.12(115.02)$ & $156.25(218.74)$ & $39.06(7.20)$ \\
\hline$K$. pneumoniae & $19.53(28.75)$ & $78.12(109.37)$ & $19.53(3.60)$ & $39.06(57.51)$ & $156.25(218.74)$ & $39.06(7.20)$ \\
\hline S. typhi & $19.53(28.75)$ & $39.06(54.68)$ & $19.53(3.60)$ & $39.06(57.51)$ & $78.12(109.37)$ & $39.06(7.20)$ \\
\hline \multicolumn{7}{|c|}{ Gram-positive bacteria } \\
\hline B. cereus & $9.76(14.37)$ & $39.06(54.68)$ & $2.44(0.45)$ & $39.06(57.51)$ & $78.12(109.37)$ & $4.88(0.90)$ \\
\hline S. aureus & $39.06(57.51)$ & $156.25(218.74)$ & $9.76(1.80)$ & $78.12(115.02)$ & $>156.25(>218.74)$ & $19.53(3.60)$ \\
\hline S. faecalis & $9.76(14.37)$ & $19.53(27.34)$ & $4.88(0.90)$ & $39.06(57.51)$ & $78.12(109.37)$ & $9.76(1.80)$ \\
\hline \multicolumn{7}{|l|}{ Fungi } \\
\hline C. albicans & $39.06(57.51)$ & $>156.25(>218.74)$ & $9.76(1.04)$ & $78.12(115.02)$ & $>156.25(>218.74)$ & $19.53(2.08)$ \\
\hline M. audouinii & $156.25(230.03)$ & $39.06(54.68)$ & $9.76(1.04)$ & $>156.25(>230.03)$ & $156.25(218.74)$ & $19.53(2.08)$ \\
\hline
\end{tabular}

a) RA: reference antibiotics (gentamicin for bacteria and nystatin for fungi). 
tive ion mode on an API QSTAR pulsar mass spectrometer.

Analytical thin layer chromatography (TLC) was performed on Kieselgel $60 \mathrm{~F}_{254}$ precoated Al sheet $(0.2 \mathrm{~mm}$ layer thickness, Merck) and the spots were detected by ultraviolet irradiation $(254,366 \mathrm{~nm})$ and by spraying with $10 \% \mathrm{H}_{2} \mathrm{SO}_{4}$ reagent.

Plant Material The roots of Psorospermum febrifugum were collected at Bantoum, West Province (Cameroon), in September 2004, while the barks of Psorospermum adamauense were harvested at Batsenga, Centre Province (Cameroon), in March 2007. These two plants were identified by Mr. Nana (plant taxonomist) of National Herbarium, Yaounde where voucher specimens of $P$. febrifugum $\left(\mathrm{N}^{\circ} 9826 \mathrm{SRF}\right.$ Cam) and $P$. adamauense $\left(\mathrm{N}^{\circ}\right.$ 41149/HNC) documenting the collection, have been deposited.

Extraction and Isolation Air-dried powders roots of $P$. febrifugum $(4 \mathrm{~kg})$ and bark of $P$. adamauense $(7 \mathrm{~kg})$ were separately extracted by maceration at room temperature for $48 \mathrm{~h}$ using a methanol/dichloromethane $(1: 1)$ mixture. The suspensions were filtered and each filtrate was concentrated under reduced pressure to give $300 \mathrm{~g}$ and $580 \mathrm{~g}$ of crude residue, respectively. The crude extract from the roots of P. febrifugum $(300 \mathrm{~g})$ were subjected to flash column chromatography on silica gel (Merck, 230-400 mesh) and eluted with hexane, hexane/AcOEt (4:1), hexane/AcOEt (1:1), hexane/AcOEt (1:4), AcOEt to give five fractions labelled $\mathrm{F}_{1}(53 \mathrm{~g}), \mathrm{F}_{2}$ $(40 \mathrm{~g}), \mathrm{F}_{3}(25 \mathrm{~g}), \mathrm{F}_{4}(12 \mathrm{~g}), \mathrm{F}_{5}(10 \mathrm{~g})$. Fractions $\mathrm{F}_{1}$ and $\mathrm{F}_{2}$ were mixed, on the basis of their thin layer chromatography (TLC). Fraction F (93 g) obtained was further subjected to column chromatography (CC) on silica gel (Merck, $70-230 \mathrm{mesh}$ ) and eluted with hexane/AcOEt mixture of increasing polarity. One hundred and twenty fractions of $150 \mathrm{ml}$ each were collected and analysed by TLC using hexane/AcOEt $(7: 3)$ as mobile phase. Sub-fractions $2-10,11-20,99-101$ and 104-120 were left to crystallize at room temperature to afford, respectively: 3-geranyloxyemodin anthrone (30 mg), bianthrone $\mathrm{A}_{1}$ (3) (40 mg), febrifuquinone (1) (8 mg) and vismione D (4) $(20 \mathrm{mg})$. Similarly, the crude extract of the barks of $P$. adamauense $(300 \mathrm{~g})$ were also subjected to flash column chromatography on silica gel (Merck, 230-400 mesh) and eluted with hexane, hexane/AcOEt (4:1), hexane/AcOEt $(1: 1)$, hexane/AcOEt $(1: 4)$, AcOEt to give five fractions labelled $A_{1}(52 \mathrm{~g}), \mathrm{A}_{2}(80 \mathrm{~g}), \mathrm{A}_{3}(7.1 \mathrm{~g}), \mathrm{A}_{4}(8 \mathrm{~g}), \mathrm{A}_{5}(10 \mathrm{~g})$. Fractions $\mathrm{A}_{1}$ and $\mathrm{A}_{2}$ were also mixed, according to the spots observed on their TLC. Fraction $\mathrm{F}(93 \mathrm{~g}) \mathrm{A}_{1}$ and $\mathrm{A}_{2}$ were also mixed on the basis of TLC. We obtained fraction A (132 g) which was treated as fraction F. Two hundred and thirty-one fractions of $150 \mathrm{ml}$ each were collected and analysed by TLC using hexane/AcOEt $(19: 1)$ as mobile phase. Sub-fractions 39-56, 97-109, $132-144$ and $161-170$, also left at room temperature, were crystallized and gave, respectively: 3-geranyloxyemodin anthrone (80 mg); 1,8-dihydroxy-3-isoprenyloxy-6-methylanthraquinone $(20 \mathrm{mg})$; bianthrone $\mathrm{A}_{2 b}$ (25 mg); 2-geranylemodin (20 mg) and emodin (5) (14 mg). The residues obtained from sub-fractions 39-59 and 153-170 and labelled $\mathrm{A}^{\prime}$ and $\mathrm{A}^{\prime \prime}$, were eluted, respectively with the mixture of hexane/AcOEt $(9: 1)$ and of hexane/AcOEt $(19: 1)$ and afforded 3-geranyloxy-1,8-dihydroxy-6-methylanthraquinone $(20 \mathrm{mg})$ and adamabianthrone $(2)(6 \mathrm{mg})$, respectively.

Febrifuquinone (1): Red powder. mp $132-133^{\circ} \mathrm{C}$. UV $\lambda_{\max }(\mathrm{EtOH}) \mathrm{nm}$ : 235, 320, 390 and 435. IR (KBr) cm $\mathrm{cm}^{-1}: 3630,3360,3300,3050,3020,2860$, 1668, 1640, 1630, 1560, 1110. ${ }^{1} \mathrm{H}-$ and ${ }^{13} \mathrm{C}-\mathrm{NMR}$ (see Table 1). HR-ESITOF-MS $m / z: 679[\mathrm{M}+\mathrm{H}]^{+}$. HR-ESI-TOF-MS m/z: 679.2514 (Calcd for $\mathrm{C}_{40} \mathrm{H}_{39} \mathrm{O}_{10}:$ 679.2532). HR-ESI-TOF-MS/MS m/z: $409\left[\mathrm{M}-\mathrm{C}_{15} \mathrm{H}_{9} \mathrm{O}_{5}\right]^{+}, 269$ $\left[\mathrm{M}-\mathrm{C}_{25} \mathrm{H}_{29} \mathrm{O}_{5}\right]^{+}, 525\left[\mathrm{M}-\mathrm{C}_{10} \mathrm{H}_{17} \mathrm{O}\right]^{+}$

Adamabianthrone (2): Yellow powder. mp $152-154^{\circ} \mathrm{C}$. UV $\lambda_{\max }(\mathrm{EtOH})$ $\mathrm{nm}: 320,270,363$. IR (KBr) $\mathrm{cm}^{-1}: 3620,3366,3040,2870,2870,1630$, 1565, 1100. ${ }^{1} \mathrm{H}$ - and ${ }^{13} \mathrm{C}-\mathrm{NMR}$ (see Table 2). HR-ESI-TOF-MS $\mathrm{m} / \mathrm{z}: 715$ $[\mathrm{M}+\mathrm{H}]^{+}$. HR-ESI-TOF-MS $m / z: 715.3231$ (Calcd for $\mathrm{C}_{45} \mathrm{H}_{47} \mathrm{O}_{8}:$ 715.3258). HR-ESI-TOF-MS/MS m/z: $391\left[\mathrm{M}-\mathrm{C}_{20} \mathrm{H}_{19} \mathrm{O}_{4}\right]^{+}, 323\left[\mathrm{M}-\mathrm{C}_{25} \mathrm{H}_{27} \mathrm{O}_{4}\right]^{+}, 561$ $\left[\mathrm{M}-\mathrm{C}_{10} \mathrm{H}_{17} \mathrm{O}\right]^{+}$.

Antimicrobial Assays. Microbial Strains The microorganisms tested included Bacillus cereus LMP0404G, Streptococcus faecalis LMP0207U, methicillin-resistant Staphylococcus aureus LMP805, as three Gram-positive bacteria, five Gram-negative bacteria namely $\beta$-lactamase positive $(\beta \mathrm{L}+)$ Escherichia coli LMP701, ampicillin-resistant Klebsiella pneumoniae LMP803, carbenicillin-resistant Pseudomonas aeruginosa LMP804, chloramphenicol-resistant Salmonella typhi LMP706, chloramphenicol-resistant Citrobacter freundii LMP802 and two fungi namely Candida albicans LMP709U, Microsporum audouinii LMP725D. These microbial species are clinical isolated from patients in the Yaounde General Hospital (Cameroon).

Their identification were confirmed before usage in the Laboratory of Applied Microbiology and Molecular Pharmacology (LMP) (Faculty of Science, University of Yaoundé I) where the antimicrobial tests were per- formed, using API system as previously reported. ${ }^{26)}$ They were maintained on agar slant at $4{ }^{\circ} \mathrm{C}$ at the LMP and sub-cultured on a fresh appropriate agar plate for $24-48 \mathrm{~h}$ prior to any antimicrobial test.

MIC and MMC Determinations: Microdilution Method The MICs of compounds 1, 2 and reference antibiotics (RA) (gentamicin for bacteria and nystatin for fungi) were evaluated as follows: the tested samples were first of all dissolved in dimethylsulfoxide (DMSO). The solution obtained was added to muller hinton agar (MHA) to give a final concentration of $156.25 \mu \mathrm{g} / \mathrm{ml}$. This was serially diluted two fold to obtain concentration ranged from 1.22 to $156.25 \mu \mathrm{g} / \mathrm{ml}$. One hundred microliters of each concentration was added in a well (96-wells microplate) containing $95 \mu \mathrm{l}$ of nutrient broth containing $0.05 \%$ phenol red and supplemented with $10 \%$ glucose (NBGP) and $5 \mu \mathrm{l}$ of inoculum [(at $1.5 \times 10^{6}$ colony forming unit (CFU) $/ \mathrm{ml}$ by adjusting the optical density to 0.1 at $600 \mathrm{~nm}$ (SHIMADZU UV-120-01 spectrophotometer)]. ${ }^{21-25)}$ The final concentration of DMSO in the well was less than $1 \%$ (preliminary analyses with $1 \%(\mathrm{v} / \mathrm{v})$ DMSO/NBGP do not show the growth of the tested microorganisms). The negative control well consisted of $195 \mu \mathrm{l}$ of NBGP and $5 \mu \mathrm{l}$ of the standard inoculum. ${ }^{21-25)}$ The plates were covered with a sterile plate sealer, then agitated to mix the content of the wells using a plate shaker and incubated at $37^{\circ} \mathrm{C}$ for $24 \mathrm{~h}$. The assay was repeated twice. The MIC of samples was detected following addition $(40 \mu \mathrm{l})$ of $0.2 \mathrm{mg} / \mathrm{ml} p$-iodonitrotetrazolium chloride and incubated at $37^{\circ} \mathrm{C}$ for $30 \mathrm{~min} .{ }^{25}$ Viable bacteria reduced the yellow dye to a pink colour. The lowest concentration showing no colour change was considered as the MIC. For the determination of MMC, a portion of liquid $(5 \mu \mathrm{l})$ from each well that showed no change in colour was placed on MHA and incubated at $37^{\circ} \mathrm{C}$ for $24 \mathrm{~h}$. The lowest concentration that yielded no growth after this sub-culturing was taken as the MMC. ${ }^{21-25)}$

Acknowledgements This investigation was supported by TWAS, The Academy of Sciences for the Developing World c/o ICTP-Strada Costiera 11 34014 Trieste, Italy, through Grant No. 07-141 LDC/CHE/AF/AC-UNESCO FR awarded to Professors Tsamo and Nkengfack's Labs, respectively.

\section{References}

1) Perrier de Labathie H., "Flore de Madagascar et des Comores (Plantes vasculaires)," Typographie Firmin-Didot et Cie, 56-rue Jacob, Paris, 1950, pp. $10-14$.

2) Uphof J. C. Th., "Dictionary of Economic Plants," 2nd ed., Strechert Hafner Service Agency Inc., New York, 1968, p. 434.

3) Watt J. M., Brayer-Brandwijk M. G., "The Medicinal and Poisonous Plants of Southern and Eastern Africa," 2nd ed., E and S, Livingstone Ltd., Edinburgh, 1962, pp. 494-500.

4) Irvine F. R., "Woody Plants of Ghana," Oxford University Press, London, 1961, pp. 140-143.

5) Botta B., Monache F. D., Monache G. D., Bettolo G. B. M., Oguakwa J. U., Phytochemistry, 22, 539-542 (1983).

6) Monache G. D., Monache F. D., Di Benedetto R., Oguakwa J. U., Phytochemistry, 26, 2611-2613 (1987).

7) Monache F. D., Botta B., Monache G. D., Bettolo G. B. M., Phytochemistry, 24, 1855-1856 (1985).

8) Botta B., Monache F. D., Monache G. D., Bettolo G. B. M., Kabangu K., Phytochemistry, 25, 766 (1986).

9) Marston A., Chapuis J. C., Sordat B., Msonthi J. D., Hostettman K., Planta Med., 207-210 (1986).

10) Botta B., Monache F. D., Monache G. D., Bettolo G. B. M., Msonthi J. D., Phytochemistry, 24, 827-830 (1985).

11) Amonkar A., Chang C.-J., Cassadi J. M., Experientia, 37, 1138-1140 (1981).

12) Botta B., Monache F. D., Monache G. D., Tetrahedron Lett., 28, 567571 (1987).

13) Monache F. D., Torres F. F., Bettolo M. G. B., de Lima A., J. Nat. Prod., 43, 487-494 (1980).

14) Kupchan S. M., Streelman D. R., Sneden A. T., J. Nat. Prod., 43, 296-301 (1980)

15) Abou-Shoer M., Boettner F. E., Chang C.-J., Cassadi M. J., Phytochemistry, 27, 2795-2800 (1988).

16) Abou-Shoer M., Suwanborirux K., Habib A. A. M., Chang C. J., Cassady J. M., Phytochemistry, 34, 1413-1420 (1993).

17) Cohen P. A., Towers G. H. N., J. Nat. Prod., 58, 520-526 (1995).

18) Camelle G., Monache F. D., Monache G. D., Bettolo G. B. M., Alves De Lima R., Phytochemistry, 21, 417-419 (1982).

19) Bilia A. R., Yusuf A. W., Braca A., Keita A., Morelli I., J. Nat. Prod., 63, 16-21 (2000). 
20) Kouam S. F., Ngadjui B. T., Krohn K., Wafo P., Ajaz A., Choudhary M. I., Phytochemistry, 66, 1174-1179 (2005).

21) Kuete V., Nguemeving J. R., Penlap Beng V., Azebaze A. G. B., Etoa F.-X., Meyer M., Bodo B., Nkengfack A. E., J. Ethnopharm., 109, 372-379 (2007a).

22) Kuete V., Konga Simo I., Ngameni B., Bigoga D. J., Watchueng J., Nzesse Kapguep R., Etoa F.-X., Ngadjui T. B., Penlap B. V., J. Ethnopharm., 112, 271-277 (2007b).

23) Kuete V., Eyong K. O., Folefoc G. N., Beng V. P., Hussain H., Krohn K., Nkengfack A. E., Pharmazie, 62, 552-556 (2007c).

24) Kuete V., Wansi J. D., Mbaveng T. A., Kana Sop M. M., Tcho Tadjong
A., Beng P. V., Etoa F.-X., Wandji J., Meyer J. J. M., Lall N., South African Journal of Botany, 10.1016/j.sajb.2008.02.004 (2008a).

25) Kuete V., Mbaveng T. A., Tsaffack M., Beng P. V., Etoa F.-X., Nkengfack A. E., Meyer J. J. M., Lall N., J. Ethnopharm., 115, 494-501 (2008b).

26) Mbaveng A. T., Ngameni B., Kuete V., Konga S. I., Ambassa T., Roy R., Bezabih M., Etoa F.-X., Ngadjui B. T., Abegaz B. M., Meyer J. J. M., Lall N., Penlap B. V., J. Ethnopharm., $483-489$ (2008).

27) Mbwambo Z. H., Apers S., Moshi M. J., Kapingu M. C., Van Miert S., Claeys M., Brun R., Cos P., Pieters L., Vlietinck A., Planta Med., 70, $706-710$ (2004). 